\title{
MOTOR RESPONSES FROM THE MACAQUE OCCIPITAL LOBE ${ }^{1}$
}

\author{
NORMAN W. RIECK ${ }^{2}$ \\ Laboratory of Comparative Neurology, Department of Anatomy, \\ University of Michigan, Ann Arbor \\ THIRTEEN FIGURES \\ INTRODUCTION
}

There is considerable interest at the present time in certain cortical areas, other than those bordering the central fissure, which yield various types of eye, head, body, and extremity movements on stimulation. Travis ('55) and others showed that for monkeys - and presumably the same condition exists for man - these areas function in maintaining the normal posture of the body. They also are concerned in supplementing and enriching voluntary movements. They are called, then, supplementary or additional or second motor areas. Aside from occasional awkward posturing - and this is not always noticeable - the loss of one of these supplementary areas produces no evident motor deficit. However, as one after the other of these areas is ablated, there is an increased poverty of movement, which becomes marked when the motor cortex has been destroyed, particularly bilaterally. It seems probable that these additional motor areas are trigger points for the initiation of Jacksonian convulsions.

The present study is concerned with supplementary motor areas demonstrable in the occipital lobe of the macaque. It

${ }^{1}$ A dissertation submitted in partial fulfillment of the requirements for the degree of Doctor of Philosophy in the University of Michigan, 1957.

${ }^{2}$ Present address: Temple University School of Medicine, Philadelphia, Pa. 
also considers some of the pathways over which discharge is made from this lobe to brain stem centers.

\section{MATERIALS AND METHODS}

The animals used in this study were macaques (Macaca mulatta). All of the monkeys chosen appeared to be strong, alert, and healthy prior to the onset of the experimental work. Eight monkeys representing 12 experiments formed the basis of this study. Aseptic surgical technique was employed in all experiments. From $0.15-0.20 \mathrm{~cm}^{3}$ of atropine sulfate was administered to all but the first monkey of the series studied, before each animal was anesthetized. Stimulation of the cerebral cortex was carried on while the animals were under light ether anesthesia. The Grass stimulator (model 3C) was used for all cortical excitation as well as for some of the cortical destruction. A frequency of 40 vibrations per second and a strength of stimulus varying between 4 and 7 volts were employed. Duration of the square wave pulses was one millisecond. An insulated unipolar platinum electrode was used to stimulate as well as to cauterize the cortex. Lesions were made either by cauterizing or by removing small areas of cortical tissue by suction, and sometimes by a combination of the two methods. Gelfoam was placed in the larger lesions. In order to prevent the animals from carrying infection into their wounds, a small piece of cotton was sewed over the incision and then covered with celloidin. Postoperatively the monkeys were observed for motor and/or visual defects.

Each monkey was sacrificed between the 14th and 21st day after completion of the second experiment, or between the 14th and 21st postoperative day where only one surgical procedure was performed. The arterial system was perfused with $500-1000 \mathrm{~cm}^{3}$ of $10 \%$ formalin after an overdose of ether or sodium pentothal had been administered. The brain was then carefully removed and placed in $10 \%$ formalin for a period of not less than 14 days prior to staining. Each brain was prepared by the Marchi technique using a modification of the Swank and Davenport ('35) method. 


\section{PERTINENT LITERATURE}

Over the years a very considerable literature dealing with supplementary motor areas has accumulated. As a background for the material to be reported upon in the present paper, a brief review of some of the related studies on the primate supplementary or additional motor areas will be presented.

Foerster ('31), Penfield and Welch ('51), and Travis ('55) have demonstrated a pattern for second motor area responses from area 6. Foerster's responses were largely of a type of avoiding movements since they consisted of a turning of the head, body, and eyes away from the side stimulated. He demonstrated his pattern of responses in both monkey and man, largely from a portion of area 6 on the lateral surface of the hemisphere. Penfield and Welch obtained a series of movements on stimulation of area 6 on the medial portion of the hemisphere in the monkey. Their pattern showed considerable overlapping between upper extremity and lower extremity areas. In general, the regions giving lower extremity responses were caudal to those producing upper extremity movements on suitable stimulation, and bordered the "foot" and "leg", areas of the motor cortex. The pattern described by Travis for the monkey was demonstrable on area 6 on both the medial and the lateral surfaces of the hemisphere and was relatively discrete. This observer obtained lower extremity movements from regions adjoining the motor cortex, followed rostrally by upper extremity. movements and then by head movements. She pointed out the significance of area 6 for the maintenance of normal posture.

The elicitation of eye deviations in several planes by stimulation of frontal lobe areas in the monkey has been recognized by many observers (Ferrier, 1876; Ferrier and Yeo, 1884 ; Horsley and Schäfer, 1888; Sherrington, 1893; Smith, '40 and '49; Crosby, Yoss, and Henderson, '52 and many others). Foerster ('31), Penfield and Boldrey ('37), Penfield and Erickson ('41), Penfield and Rasmussen ('50), and 
others have demonstrated such deviations in man on similar stimulation. In addition to the primary motor field for eye movements demonstrable on stimulation from the region of the frontal lobe of the macaque dorsal to the principal fissure and rostral to the arcuate fissure, a supplementary motor eye field ventral to this fissure and rostral to the primary field, and with a mirror-image arrangement of its constituents, has been described (Crosby, Yoss, and Henderson, '52; Crosby, '53).

An additional motor field has been demonstrated in the parietal areas 5 and 7 of the monkey by Peele ('44) and by Fleming and Crosby ('55). Stimulation of area 5 in the most dorsal regions of the lateral hemisphere wall produced movements of both lower extremities. Ventral to this, along the rostral border of the intraparietal fissure, gross movements of the upper extremity were obtained on stimulation. Still farther ventralward, contralateral face movements and, at the base of the intraparietal fissure, homolateral sneering and closing of the eye were produced on excitation. Some of these movements could also be elicited from the rostral end of area 7 immediately behind the intraparietal fissure. From the most caudal portions of area 7 , bordering on area 19 , eye deviations were obtained.

From the preoccipital areas of the cortex a very definite pattern of motor responses is elicitable on stimulation when the animal is under light (ether) anesthesia. Foerster ('31) obtained, in a patient, conjugate horizontal deviation of the eyes toward the side opposite excitation from stimulation of the preoccipital area. The most complete patterns have been given by Crosby and Henderson ('48) and Lemmen ('51). The upper part of the preoccipital field is concerned, in general, with deviation of the eyes. Most dorsally, conjugate deviation of the eyes upward toward the other side (directly or obliquely) is obtainable. Immediately adjacent to this region, on the lateral hemisphere wall, is an area from which conjugate deviation of the eyes toward the side opposite stimulation can be demonstrated. Then follows a region, 
stimulation of which gives obliquely downward and then directly downward deviation of the eyes. Beneath the area from which eye deviations are elicitable, and so in the lower half of the preoccipital field, Lemmen ('51) obtained, in dorsoventral arrangement on stimulation of this cortical area in the monkey, homolateral face responses, then contralateral face movements and, most ventrally, contralateral or bilateral arm movements. Leg movements are obtainable from the ventral surface of the brain at the caudalmost part of the temporal area.

This preoccipital area is concerned with following eye movements, fixation of the eyes, and with body movements, primarily in response to visual excitation. Irritative lesions in the portions of the preoccipital and occipital areas concerned with deviations may produce optokinetic nystagmus (Henderson and Crosby, '52). The literature dealing with movements evoked by stimulation of the occipital areas of the macaque by Walker and Weaver ('40), Crosby and Henderson ('48), Crosby ('53), and others is considered in the discussion of the present paper and will not be reviewed here.

From the tip of the temporal lobe to the occipital region of the hemisphere, across the middle and inferior temporal gyri, a second or additional motor area has been demonstrated in the monkey. On stimulation of the temporal pole, homolateral face movements have been obtained by Schneider and Crosby ('54) for monkey and by Baldwin, Frost, and Wood ('54 and '56) for primates, including man. This pattern for homolateral face movements has been extended occipitalward through the inferior temporal gyrus by $B$. DeJonge (paper to be published). Ventral to the strip related to homolateral face movement, throughout the caudal twothirds of the temporal area, there is a region concerned with contralateral responses of the face. Still farther ventralward, along the lower part of the lateral temporal wall. homolateral and then contralateral upper extremity movements are elicitable on proper stimulation, with lower extremity movements represented on the under side of the 
temporal lobe (B. DeJonge). The extension of these areas into the occipital lobe is indicated in the protocols and considered in the discussion of the present paper.

A supplementary motor area, area 22 , has been demonstrated in the superior temporal gyrus by Foerster ('31) for man and by Lemmen ('51) for the monkey. The movements obtained from area 22 are in the nature of avoiding reactions.

In relation to the island, two distinct motor patterns have been demonstrated. The more dorsal of these begins with the region in the inferior frontal area from which ipsilateral face movements and, slightly farther occipitalward, contralateral face movements can be obtained on stimulation. Arm movements are produced from stimulation of the parietal operculum near the region of the central fissure and leg movements from the dorsocaudal part of the island. Another second or additional motor pattern demonstrable across the island extends over its ventral half and has an arrangement similar to that in the dorsal half. Thus, on stimulation, the rostral end of the island gives homolateral face movements and the most caudal part yields lower extremity movements, usually bilaterally. Between these points the pattern is represented rostrocaudally by areas that give rise to contralateral face responses and movements of the contralateral upper extremity (and sometimes of both upper extremities). The pattern for the dorsal part of the island was demonstrated by Chusid, Sugar and French ('48); that for the ventral part was reported by Frontera ('56), who, however, questioned the reliability and constancy of its appearance. It has been redemonstrated by Showers and Lauer in a paper soon to appear.

Recently Votaw ('57) has obtained motor responses on stimulation of the hippocampus. His results suggest that this cortical area has a definite efferent pattern which probably functions in emotional expression. Stimulation of a considerable portion of the hippocampus, beginning with the pes hippocampi, is followed by face movements - homolat- 
eral, bilateral, or contralateral - depending upon the exact point of stimulation. Stimulation of the caudodorsal portion of the hippocampus gives upper extremity movements but lower extremity movements, curiously enough, were not obtained by stimulation of this cortical area except as a part of a general awakening or mass movement. A detailed account of Dr. Votaw's work on hippocampus is to appear shortly.

The discharge paths to lower centers from the various additional motor areas are by no means completely known. Some of the regions, notably the frontal eye fields and area 5 , contribute fibers to the pyramidal system, although they have other significant connections. Area 5 also sends fibers to the putamen (Crosby, '56). Area 6 discharges to the globus pallidus and to the putamen by corticostriate paths (Dusser de Barenne, Garol, and McCulloch, '41; Crosby, '56). It is possible that there are also direct corticotegmental bundles from this area. The temporal areas relay partly through the basal ganglia (Crosby, '56) and partly by direct. corticotegmental paths (Wall and Davis, '51). The preoccipital and occipital areas have been shown to project to the superior colliculus (Crosby and Henderson, '48; Peterson and Henneman, '48; Lemmen, '51) and to the tegmentum of the midbrain (Crosby and Henderson, '48; Lemmen, '51) where synapses are made with pathways to appropriate motor centers. The major hippocampal discharge is by way of the fornix (Votaw, '57). It is to be stressed that many of these discharge paths have courses distinct from that of the pyramidal system and so may function when this latter system has been destroyed.

It is evident that the supplementary motor areas of the primate cortex are numerous and that the patterns of movements elicitable from them are strikingly similar in arrangement. These patterns resemble each other also in appearing, in all cases, as gross movements. Very delicate individual movements imply an intact motor cortex and an uninjured pyramidal system. In view of the findings in other areas of 
the cortex it is not surprising that we have been able to demonstrate a pattern of additional motor movements on the occipital lobe.

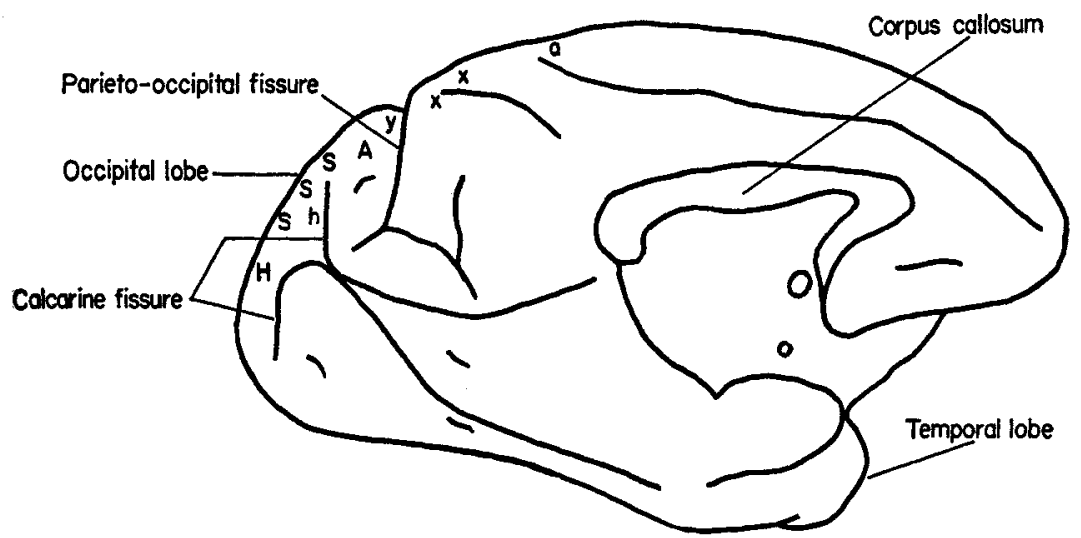

Fig. 1. A diagram of the medial surface of the left hemisphere of Macaca mulatta. The movements resulting from stimulation at various points are indicated as follows: a, ipsilateral forearm; $x$, ipsilateral upper arm; $y$, upper arm and neck; A, ipsilateral arm; S, bilateral shoulders; $h$, head turning toward side opposite the stimulus; $\mathrm{H}$, head turning toward the side of the stimulus.

\section{RESULTS OF EXPERIMENTAL WORK}

\section{Monkey $R 2$}

\section{Experimental work}

On February 25, 1955, a right parieto-occipital craniotomy was performed on a male Macaca mulatta. The lateral surfaces of the parietal and occipital lobes were exposed.

The medial surface of the occipital lobe was stimulated by inserting a unipolar electrode through the lateral wall until it touched the falx cerebri and then withdrawing it slightly. At a strength of stimulus which varied between 5 and 7 volts, gross movements of the ipsilateral arm were elicited. A lesion was placed at the point of stimulation by using 300 ma of eurrent for 15 seconds. The dura, the subcutaneous tissues, and finally the skin were closed using interrupted stitches.

Postoperative observations revealed a bilateral tremor in the shoulders, the upper arms and the head (including the ears), and, rarely, in the legs. There were also occasional jerky movements of the head. For the most part, the animal maintained a very apathetic attitude. However, he was capable of rapid, agile movement when this was 
expedient. These postoperative phenomena are believed to have been due to a vaseular accident which the animal suffered during surgery and not to have been a result of the cortical lesion.

On March 18, 1955, this animal was again prepared for aseptic surgery. A left parieto-occipital craniotomy was performed and the lateral surface of the parietal and occipital lobes exposed. The hemisphere was gently retracted away from the falx and the medial surface stimulated directly at voltages from $4 \frac{1}{2}$ to 7 .

From the area immediately caudal to the parieto-occipital fissure, gross movements of the upper arm and neck were elicited. Rostral to this fissure, ipsilateral upper arm movements were obtained. Moving caudalward, near the junction of the lateral and medial surfaces of the hemisphere, bilateral shoulder movements were demonstrated from all points stimulated (fig. 1).

The animal was sacrificed on March 18, 1955. The brain was prepared for the Marchi technique and the right hemisphere was cut parasagittally at a thickness of $50 \mu$.

\section{Anatomical study}

Marchi preparations show a bundle of fibers starting at the lesion which extends forward toward the ventricle and passes beneath it (and close to it) to a position caudal to the lenticular nucleus where it lies in the posterior part of the internal capsule. The fascicles arch downward through the postlenticular region, and there gradually curve caudalward over the most caudal end of the diencephalon to enter the midbrain tegmentum, lateral to the red nucleus (figs. 8-11). In this position the fibers gradually disappear, presumably by synapse with the tegmental gray. Another bundle of fibers ean be followed from the occipital lobe into the frontal region of the hemisphere, to a position ventral to the dorsal ramus of the arcuate fissure where area 8 is situated. This bundle is a component of the superior frontooccipital (association) fasciculus (figs. 4, 5).

\section{Monkey $R 3$}

Experimental work

A female Macaca mulatta was anesthetized with ether and a right parieto-occipital eraniotomy was performed on March 11, 1955. As the dural flap was turned a vascular injury occurred. Bleeding was controlled with Gelfoam and the bone was removed across the superior sagittal sinus.

The medial surface of the left occipital lobe was stimulated by inserting a unipolar electrode through the lateral wall of the cortex until it touched the falx cerebri and then withdrawing slightly. At 
6 volts, ipsilateral gross arm movements were obtained. This area was then removed by suction and Gelfoam was placed in the wound. The dura and subcutaneous tissues were closed using interrupted stitches, and the skin incision was closed with a continuous suture.

The day after operation there were no abnormalities observed. Three days postoperatively a mild tremor across the shoulders was noted. This, however, proved to be transient and the remainder of the postoperative course was uneventful. On March 31, 1955 the animal was sacrificed.

\section{Anatomical study}

The brain was cut transversely in $50 \mu$ sections. These sections reveal some degenerated fibers which extend forward from the lesion to a position underneath the ventricle, and a larger, more densely stained group which crosses to the opposite side in the corpus callosum. This latter group of fibers turns down in the posterior limb of the internal capsule and small, scattered fascicles are found in the tegmentum of the midbrain in the region lateral to the red nucleus (figs. 6-13).

\section{Monkey R4}

\section{Experimental work}

On April 15, 1955, a right parieto-occipital craniotomy was performed on a quiet, easily handled male Macaca mulatta. The hemisphere was gently retracted from the falx cerebri after the dural flap had been turned.

The medial surface of the parietal lobe just caudal to the plane of the central fissure (which represents another additional motor area in monkey) was stimulated at voltages which varied from 4-6 volts. At the higher voltage, bilateral shoulder movements obscured any other responses. At the lower voltage, ipsilateral forearm movements were obtained. These movements consisted of so-called "running" movements of the forearm and supination at the elbow (fig. 1a). A small amount of cortical tissue was removed by suction from the area previously stimulated. The dura, subcutaneous tissues, and skin were closed in the usual manner and a cotton bandage was sewed over the incision and covered with celloidin in order to keep the wound clean.

For the first few days after surgery there was no visible defect. On the 5th postoperative day a slight paresis of the left lower extremity was noted when the animal was running. This defect remained throughout the postoperative course. Moreover, the monkey held the left lower limb in an abnormal position while at rest and 
did not grasp well with the left foot. The animal appeared to be more aggressive than he had been prior to surgery.

On April 22, 1955, the animal was prepared for a second aseptic surgical procedure. A left occipital craniotomy was performed. A large cranial opening was made and, on turning the dural flap, the occipital pole could be seen. The hemisphere was retracted from the falx cerebri and stimulated at various points using a unipolar electrode.

Bilateral shoulder movements were obtained near the junction of the lateral and medial surfaces of the hemisphere, just caudal to the parieto-occipital fissure. Ipsilateral gross arm movements were produced from a small area on the medial surface of the hemisphere, slightly more caudal and more ventral than the previous site of excitation (fig. 1, X). Stimulation at a point just behind the dorsal ramus of the calcarine fissure resulted in a turning of the head toward the side opposite the stimulus. There was also some shoulder movement, but it was not so marked as that previously elicited. Farther caudalward, near the occipital pole, head movements toward the side of the stimulus, (fig. $1, \mathrm{H}$ ) as well as slight shoulder movements, were obtained (fig. 1, s). A lesion was placed at this last point by sucking out a small portion of the cortex. The usual procedure for closure and protection was followed. Due to the rather lengthy operation, 0.5 $\mathrm{cm}^{3}$ of penicillin was given intramuscularly as a precautionary measure.

On the 5th postoperative day the monkey appeared apathetic and drowsy. This drowsiness gradually disappeared, and an aggressive attitude of the animal was once more observed. The paresis of the left lower extremity, which was noted after the first operation, remained throughout the postoperative course. The animal was sacrificed and perfused on May 9, 1955.

\section{Anatomical study}

The brain was eut transversely and sections of $50 \mu$ thickness were made. A study of these sections shows two groups of degenerated fibers. The first, and larger, of these groups, arising from the portion of the right occipital lobe from which arm movements had been obtained on stimulation and where a lesion had been placed, crosses to the opposite side in the corpus callosum, turns downward in the posterior limb of the internal capsule, and can be found as scattered fibers through the tegmentum of the midbrain in the region lateral to the red nucleus (figs. 6, 7, 10-13).

The second group, arising from the lesion in the left occipital region, which marks the position where head movements had been 
obtained on stimulation, passes forward to a position at the lower edge of the ventricle (figs. 8 and 9 ). It then crosses the pulvinar with the visual radiations. It also reaches the midbrain tegmentum but lies a little more dorsal in the field than does the other degenerated tract. The paresis in the left leg was due to a forward extension of the lesion into area 4 on the medial surface of the hemisphere in front of the central fissure.

\section{Monkey R5}

\section{Experimental work}

On October 12, 1956, this male Macaca mulatta was prepared for aseptic surgery. A left occipital craniotomy was performed. A dural flap was turned exposing the left lateral surface of the occipital lobe. A point approximately one-third of the distance between the parietooccipital fissure and the caudal edge of the brain, and midway between the superior margin of the brain and the lateral calcarine fissure, yielded twitching of the ipsilateral eyelid accompanied by ipsilateral facial sneering when the point was stimulated. This point was cauterized electrically and the lesion was widened by suction. A piece of Gelfoam was placed in the lesion (fig. 2). The incision was closed as usual and the postoperative course was uneventful.

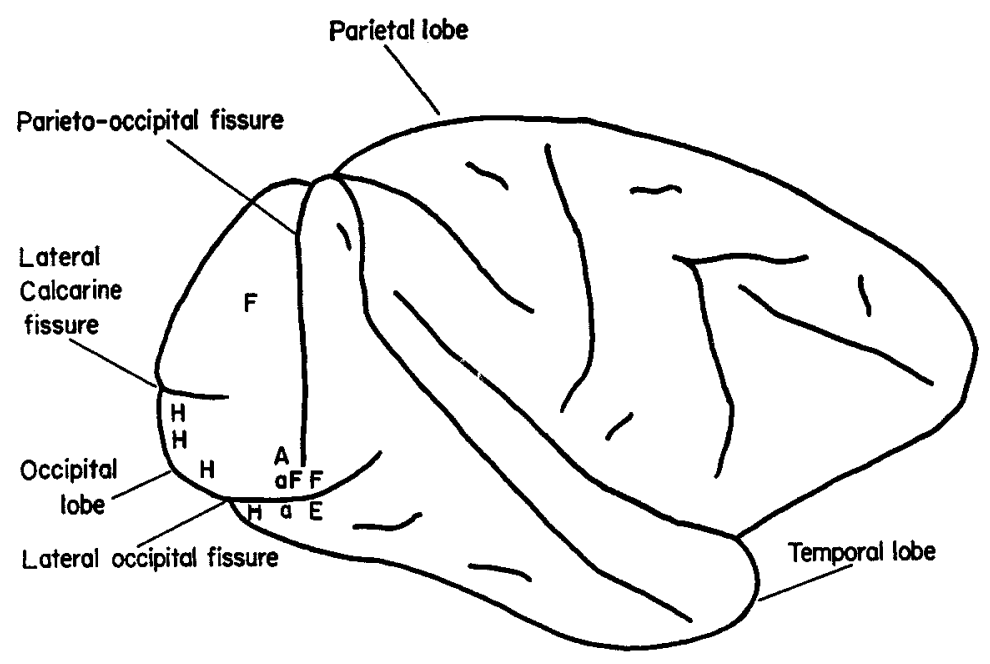

Fig. 2 A diagram of the lateral surface of the right hemisphere of Macaca mulatta. The movements resulting from stimulation of various points are indicated as follows: F, ipsilateral facial sneering and closing of the ipsilateral eyelid; $H$, head and neck turning away from the stimulus; $A$, ipsilateral arm; a, contralateral arm; E, eyes upward and toward the side of stimulation. 
On November 2, 1956, a second aseptic surgical procedure, a right occipitotemporal craniotomy, was carried out on this animal. On turning the dural flap, the ventral portion of the occipital lobe and the caudal portion of the temporal lobe were exposed. Stimulation of the middle occipital gyrus just ventral to the parieto-occipital fissure with a unipolar electrode yielded ipsilateral facial sneering and closing of the ipsilateral eyelid. A lesion was placed at this point with the electrocautery. The area of the inferior occipital gyrus just below the lateral occipital fissure yielded eye movements obliquely upward toward the side of the stimulus (fig. 2). A lesion was made in the area from which the response was obtained by cauterizing the cortex. After the lesion the eyes deviated somewhat toward the opposite side, so that they were directed obliquely upward away from the side of the previous stimulus.

The wound was closed in the usual manner. The monkey had a mild case of diarrhea at the time of the experiment, so he was given $1 \mathrm{~cm}^{3}$ of penicillin intramuscularly immediately on its completion.

The postoperative course was uneventful. The ainmal was sacrificed on November 19, 1956.

\section{Anatomical study}

The brain was prepared by the Marchi technique and eut in transverse sections of $50 \mu$ thickness. Unfortunately the material is of no value for degeneration studies because of the excessive number of artefacts throughout the sections.

\section{Monkey $R 8$}

\section{Experimental work}

On January 25, 1957, a right occipitotemporal craniotomy was performed on a male Macaca mulatta. A dural flap was turned, exposing: the inferior portion of the occipital lobe and the caudalmost portion of the temporal lobe. Stimulation of the cortex just caudal to the ventral tip of the parieto-occipital fissure yielded ipsilateral gross arm movements when the voltage was varied between $6 \frac{1}{2}$ and 7 volts. When the region immediately ventral to that just stimulated was similarly excited, contralateral gross arm movements were obtained. Continuing ventralward, a point just across the lateral occipital fissure was found which also yielded gross movements of the contralateral arm when stimulated. Caudalward, near the occipital pole, stimulation along the margin of the lateral surface caused the head and neck to turn away from the side of the excitation (fig. 2). Small lesions were placed electrically at the points from which arm move- 
ments were obtained, and the routine procedure for closure was followed. Upon recovery from the anesthesia the animal exhibited no defects and the postoperative course was uneventful.

On February 15, 1957, a left occipital craniotomy was performed exposing the most caudal and ventral portions of the occipital lobe. The unipolar electrode was bent slightly and slipped between the dura and the cortex. On the underside of the occipital pole a small area was located, stimulation of which yielded ipsilateral face movements, as previously described, and extension of the neck, with a turning of the head and neck toward the side of the stimulus. Farther rostralward a point was found from which ipsilateral face and gross arm movements, accompanied by upward and backward tilting of the head, were obtained on stimulation (fig. 3). The latter point was marked by a small lesion with the electrocautery. Routine closure followed.

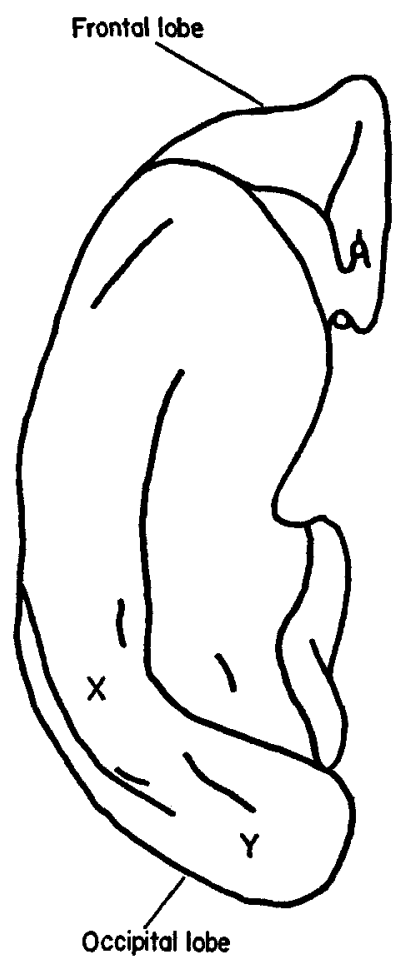

Fig. 3 A diagram of the ventral surface of the right hemisphere of Macaca mulatta. At point $\mathbf{X}$, ipsilateral face, ipsilateral arm, and head movements upward and backward were obtained on stimulation. At point $Y$, ipsilateral face, and head turning toward the side of stimulation resulted from exeitation. 
Following an uneventful postoperative course, the monkey was sacrificed on March 6, 1957. Examination of the $50 \mu$ transverse sections of the Marchi material prepared from this animal revealed that the tissue was so poorly stained that microscopic study was not feasible.

\section{DISCUSSION}

Contrary to the statement of Ferrier (1876) - "I have never obtained any reactions on stimulation of the occipital lobes" - the present experiments indicate that stimulation of this region yields a variety of types of gross head and upper extremity movements. Schäfer (1888) stimulated the "whole of the occipital lobe, including its mesial and under surfaces." He described but one result, a conjugate deviation of the eyes to the side opposite stimulation. Unfortunately, no protocols are included in his report and there is no clue as to the exact placement of the electrode during each excitation. However, he did find that stimulation in the upper portion of the occipital lobe added a downward component to the horizontal deviation, and that stimulation in the lower portion of the lobe added an upward component to the horizontal deviation. In one of our animals a point was found directly ventral to the lateral occipital fissure from which obliquely upward movements of the eyes were elicited. Similar eye movements have been found by Walker and Weaver ('40) and Crosby and Henderson ('48). The latter observers also found that stimulation dorsal to the lateral occipital fissure produced an obliquely downward deviation of the eyes.

On stimulation of the various portions of the medial surface of the occipital lobe of the macaque, we were unable to elicit eye movements. However, a small area a few millimeters caudal to the parieto-occipitial fissure consistently gave gross ipsilateral arm movements. Excitation near the place where the medial surface goes over onto the lateral surface of the hemisphere, rostral to the parieto-occipital fissure, produced ipsilateral upper arm movements. Upper arm and neck movements were obtained from the medial hemisphere wall caudal to the parieto-occipital fissure, and bilateral shoulder move- 
ments still farther caudalward. From a point just behind the dorsal ramus of the calcarine fissure, turning of the head toward the side opposite the stimulus and, from a point farther caudalward near the occipital pole, turning of the head toward the side of the stimulus were obtained on excitation.

On the lateral surface of the occipital lobe, a point was found approximately halfway between the lateral calcarine fissure and the dorsal edge of the brain, one-third of the distance from the parieto-occipital fissure and the caudal edge of the brain, from which ipsilateral facial sneering with twitching of the ipsilateral eyelid could be elicited. Farther ventralward on the lateral surface, just below the parietooccipital fissure, several points were stimulated. The result in each case was an ipsilateral facial sneering accompanied by closing of the ipsilateral eyelid. Behind the parieto-occipital fissure, points were found the excitation of which resulted in gross movement of the ipsilateral arm and similar movement of the contralateral arm (fig. 2). These facial and arm movements are in a direct line with similar movements which have been described by Schneider and Crosby ('54) and B. DeJonge (paper to be published) from stimulation of the lateral surface of the temporal lobe. Stimulation from below upward, along the caudal edge of the occipital lobe, resulted in an avoiding type of movement with the head and neck turning away from the side of excitation (fig. 2). On the tentorial surface of the occipital lobe it was found that combined movements resulted from stimulation. A point far caudalward yielded ipsilateral facial sneering with turning of the head and neck toward the side of the stimulus. Farther rostralward, a point was found from which ipsilateral face, gross ipsilateral arm, and head movements upward and backward were elicited (fig. 3). Since upward movements of the eyes are known to result from excitation of the portion of the occipital lobe along the lateral occipital fissure, it seems logical that such movements should be accompanied by a tilting of the head upward and backward. 
We have found many areas in the occipital lobe where stimulation results in movements typical of those which have been seen on excitation of other additional motor areas of the cerebral cortex. We do not regard the points discussed as small blocks of tissue which are the centers of such movements. We believe that the cortical excitations carried on in this study stimulated certain neuron ares in their course or at their region of origin and that the type of pattern and the various movements obtained in each case are determined by specific terminations of such ares on lower motor neuron groups. In this we are in agreement with Gooddy and McKissock ('51), Gooddy and Reinhold ('54), Crosby ('56), and others. Eye, face, and extremity movements similar to those described have also been obtained from the midbrain tegmentum lateral and dorsolateral to the red nucleus by Hess, Bürgi, and Bucher in the cat ('46), Crosby ('56) and Crosby, Carey, and Drew (paper to be published) in the monkey.

The lesions produced during the pursuit of this problem were small as compared to those of Mettler ('35), Lemmen ('51), and Walberg and Brodal ('53). As a consequence of these small, discrete cortical ablations, the degenerated myelinated fibers are reduced in number. The Marchi technique employed exclusively for this study of these degenerated pathways and the technical limitations of this method necessarily restrict the conclusions which can be drawn from studies in which this technique has been selected over other methods.

We have found in all cases that a bundle of degenerated fibers crosses to the opposite side in the corpus callosum and descends in the contralateral internal capsule. Walberg and Brodal ('53) have followed a similar type of degeneration in the cat after rather extensive cortical ablations. Their use of silver impregnation methods has enabled them to trace the course of such fibers for a considerable distance. The relatively large bundle of fibers is seen, in our material, to turn into the tegmentum of the midbrain where the granules become very small and diffuse in the region lateral to the red nucleus. It is suggested that this is an area of synapse with 
neurons carrying impulses from both higher and lower centers. There is another, smaller group of fibers which begins in the area of the lesion in each case and extends forward toward the lateral ventricle to pass beneath it to a position caudal to the lenticular nucleus where it joins the posterior portion of the internal capsule. It arches downward through the postlenticular region, and there gradually curves caudalward, over the most caudal end of the diencephalon, to enter the midbrain tegmentum lateral to the red nucleus, where it also gradually disappears. The fibers which have degenerated from the areas from which head movements were obtained on excitation appear to lie dorsal in the midbrain tegmentum to those from areas from which gross arm movements were elicited.

In a parasagittally cut hemisphere, a cortical association bundle can be followed from the occipital lobe into the frontal region. It reaches the position ventral to the dorsal ramus of the arcuate fissure which is occupied by area 8 .

Henderson and Crosby ('52) were able to trace (in Weil material prepared some months after the lesion was made) corticotectal fibers from the occipital and preoccipital divisions of the internal corticotectal tract to the superior colliculi. They also found a corticotegmental system from the preoccipital areas to the midbrain tegmentum dorsal and dorsolateral to the red nucleus. Neither the corticotectal fibers nor the corticotegmental fascicles described by these last mentioned observers have been identified in our material since we did not place lesions in the fields that give conjugate deviation of the eyes in various planes on stimulation. Peterson and Henneman ('48), using strychnine neuronographic techniques, found that, of all of the areas sampled on the lateral aspect of the hemisphere, only the frontal eye fields, area 19, and anterior half of the superior temporal convolution fired well into the superior colliculus. Peterson and Henneman's work confirms the Crosby and Henderson results.

The association fascicles demonstrated in our material as extending forward from the occipital cortex to the frontal lobe document the existence of a direct occipitofrontal system 
containing some fibers, at least, which do not synapse in course. Demonstration of such direct fibers has not been very frequent and some observers (for example, Bailey and Bonin, '51) have questioned whether any such direct fibers occur or whether the superior longitudinal association bundle and the superior and inferior occipitofrontal association bundles consist only of short fascicles. Possibly there are only such short fibers in some animals and the existence of longer, more direct paths is a reflection of the dominance of visual projections and visual association in primates where the eyes are so large and so important to the animal.

An example of the significance of the superior occipitofrontal association fibers is afforded by a study by Clark and Lashley ('47). These observers showed that, after destruction of the superior occipitofrontal association bundle in the monkey, the animal did not look toward an object presented in the lateral part of the visual field on the side opposite the section of the tract. Although no destruction of visual cortex had occurred, they supposed that the animal showed an homonymous hemianopsia. In reality he exhibited the pseudohomonymous hemianopsia described by certain clinical neurologists. This occurs in some frontal lobe regions where the terminal fibers of the fronto-occipital association bundle are destroyed. After such a lesion either in the association bundle or in its region of termination in the frontal cortex, a monkey (or a patient) does not lack the ability to see in the lateral visual field on the side opposite the injury, but does have an inability to respond to a visual stimulus by appropriate eye movements when connections between the visual receptive area of the occipital lobe and the motor eye center of the frontal lobe are severed.

In conclusion it may be said that our study demonstrates a specific pattern of movements elicitable from the occipital lobe which are in many respects a continuation of the pattern on the temporal lobe. The results from the different animals have been brought together in the description in the preceding pages. They are summarized in figures 1,2 , and 3 . Un- 
doubtedly these movements appear in the behavior of the normal monkey as associated movements accompanying voluntary responses, when the sensory components which set off such voluntary responses include visual stimulation and/or visual associations. The possibility that such additional motor areas may be trained to take over a considerable part of the normal functions of the motor cortex when this latter area is destroyed is of very special significance to those dealing with human cases of cerebral palsy which show diplegia or quadriplegia. A broad basis of animal experimentation is needed for possible future clinical application of the knowledge regarding such additional areas.

\section{SUMMARY}

1. A pattern of additional motor movements exists in the occipital lobe of the macaque. This pattern can be demonstrated on the medial, the lateral, and the ventral surfaces of the hemisphere in the occipital region.

2. The pattern of movements demonstrated on the lateral surface of the occipital lobe below the lateral occipital fissure is a continuation of a similar pattern known to exist in the temporal lobe.

3. It is suggested that the tilting of the head upward and backward on stimulation of the caudoventral surface of the occipital lobe is a component of the complex movement of looking upward.

4. A part of the efferent discharge is from the occipital area to the midbrain tegmentum by way of the corpus callosum and the internal capsule contralateral to the origin of the path.

5. The occipital lobe is interconnected with the primary motor eye field (area 8) by fascicles of a long association bundle, the superior occipitofrontal fasciculus.

6. The occipital lobe contributes to the overall complex reactions to sensory stimuli known as normal movement. Removal of small portions of this additional motor area in the 
macaque does not impoverish such normal movement to the extent that the deficit can be either observed or tested for by present testing methods.

7. We have been unable to produce lower extremity movement from electrical excitation of the medial, the lateral, or the ventral surfaces of the occipital lobe.

\section{ACKNOWLFDGMENTS}

The author wishes to express his sincere gratitude and appreciation to Professor Elizabeth C. Crosby whose encouragement and guidance made the execution of this study possible, and to Parke, Davis and Company, Detroit, who furnished the animals which were used for these experiments. Funds for technical assistance were provided by the Walter L. Hill Medical Research Fund and Rackham Project no. 281 of the University of Michigan.

\section{LITERATURE CITED}

BATLEY, P., AND G. V. Bonin 1951 The Isocortex of Man. University of Illinois Press, Urbana, Illinois, 301 pp.

BALdWIn, M., L. L. Frost ANd C. D. Wood 1954 Investigation of the primate amygdala: Movements of the face and jaw. Neurol., 4: 586-598.

1956 Investigation of the primate amygdala: Movements of the face and jaws. 2. Effect of selective cortical ablations. Ibid., $6: 288-293$.

Chusid, J. G., O. Sugar and J. D. French 1948 Cortico-cortical connections of the cerebral eortex lying within the arcuate and lunate sulei of the monkey (Macaca mulatta). J. Neuropath. Exp. Neurol., 7: $439-446$.

Clark, G., AND K. S. LASHLey 1947 Visual disturbance following frontal ablations in the monkey. Anat. Rec., 97: 10. (Abstract.)

CRosby, E. C. 1953 Relation of brain centers to normal and abnormal eye movements in the horizontal plane. J. Comp. Neur., 99: 437-480. (Mellon Lecture, November, 1951).

1956 The role of the midbrain as a part of the discharge path from higher centers. Progress in Neurobiology, 1: 217-231.

Crosby, E. C., AND J. W. Henderson 1948 The mammalian midbrain and isthmus regions. II. Fiber connections of the superior colliculus. B. Pathways concerned in automatic eye movements. J. Comp. Neur., 88: 53-91. 
Crosby, E. C., R. E. Yoss AND J. W. Henderson 1952 The mammalian midbrain and isthmus regions. Part II. The fiber connections. D. The pattern for eye movements on the frontal eye field and the discharge of specific portions of this field to and through midbrain levels. Ibid., 97: $357-383$.

Dusser de Barene, J. G., H. W. Garol and W. S. MeCulloch 1941 Physiological neuronography of the corticostriatal connections. Res. Publ. Assoc. Nerv. Ment. Dis., 21: 246-266.

Ferrier, D. 1876 The Functions of the Brain. Smith, Elder and Co., London, $323 \mathrm{pp}$.

FerRier, D., AND G. F. Yeo 1884 A record of experiments on the effects of lesions of different regions of the cerebral hemisphere. Philos. Trans., 175 (part II) : 479-564.

Fleming, J. F. R., And E. C. Crosby 1955 The parietal lobe as an additional motor area: The motor effects of electrical stimulation and ablations of cortical areas 5 and 7 in monkeys. J. Comp. Neur., 103: 485-512.

Foerster, O. 1931 The cerebral cortex in man. Lancet, 2: 309-312.

FrontrRA, J. 1956 Some results obtained by electrical stimulation of the cortex of the Island of Reil in the brain of the monkey (Macaca mulatta). J. Comp. Neur., 105: 365-394.

Gooddy, W., AND W. McKissock 1951 The theory of cerebral localisation. Lancet, 1: 481-483.

GOODDY, W., AND M. ReINHoLd 1954 The function of the eerebral cortex. Brain, 77 : 416-426.

HENDERSON, J. W., AND E. C. Crosby 1952 An experimental study of optokinetic responses. A.M.A. Arch. Ophthal., 47: 43-54.

Hess, W. R., S. BÜrgi ANd V. Bucher 1946 Motorische Funktion des Tektalund Tegmentalgebietes. Monatschr. Psych. Neurol., 112: 1-52.

HoRSLEY, V., AND E. A. SCHÄFER 1888 A record of experiments upon the functions of the cerebral cortex. Philos. Trans., 179B: 1-45.

IJEMMEN, L. J. 1951 An anatomical and experimental study of temporal and occipital association areas. J. Comp. Neur., 95: 521-559.

Mettuer, F. A. 1935 Corticofugal fiber connections of the cortex of Macaca mulatta. The occipital region. Ibid., 61: 221-256.

Peele, T. L. 1944 Acute and chronic parietal lobe ablations in monkeys. J. Neurophysiol., 7: 269-286.

Penfield, W. 1950 Epileptic automatism and the centrencephalic integrating system. Res. Publ. Assoc. Nerv. Ment. Dis., 30: 513.

Penfield, W., ANd E. Boldrey 1937 Somatic motor and sensory representation in the cerebral cortex of man as studied by electrical stimulation. Brain, 60: 389-443.

Penfield, W., and T. C. Erickson 1941 Epilepsy and Cerebral Localization. A study of the mechanism, treatment and prevention of epileptic seizures. Charles $\mathrm{C}$ Thomas, Springfield, Illinois, $623 \mathrm{pp}$.

Penfield, W., and T. Rasmussen 1950 The Cerebral Cortex of Man. The Macmillan Company, New York, 248 pp. 
Penfield, W., AND K. WELCH 1951 The supplementary motor area of the cerebral cortex. A clinical and experimental study. Arch, Neurol. Psych. 66: 289-317.

Peterson, E. W., AND E. Henneman 1948 Cortico-collicular connections in the macaque. Trans. Am. Neurol. Assoc., 73: 119-122.

SChNEIDER, R. C., AND E. C. Crosby 1954 Stimulation of "second" motor areas in the maeaque temporal lobe. Neurology, $4: 612-622$.

SCHÄFER, E. A. 1888 Experiments on the electrical excitation of the visual area of the cerebral cortex in the monkey. Brain, 11: 1-6.

Sherrington, C. S. 1893 Further experimental note on the correlation of action of antagonistic muscles. Proc. Roy. Soc., 5.3: 407-420.

SMITH, W. K. 1940 Electrically responsive cortex within the sulci of the frontal lobe. Anat. Rec., 76 (Suppl.): 75-76.

1949 The frontal eye field. In: The Precentral Motor Cortex, 2nd ed., ed. by P. C. Bucy. Illinois Monographs in the Mechanical Sciences, vol. IV, Urbana, Illinois. University of Illinois Press, Chap. XII, pp. 307-342.

SWANK, R. I., AND H. A. DAvenPoRT 1935 Chlorate-osmic-formalin method for staining degenerating myelin. Stain Tech., 10: 87-90.

Travis, A. M. 1955 Neurological deficiencies following supplementary motor area lesions in Macaca mulatta. Brain, 78: 174-198.

WALberg, F., AND A. BRodal 1953 Pyramidal tract fibres from temporal and occipital lobes. An experimental study in the eat. Ibid., 76: 491-508.

WALkER, A. E., AND T. A. WEAVER, JR. 1940 Ocular movements from the oecipital lobe of the monkey. J. Neurophysiol., 4: 353-357.

WALL, P. D., AND G. D. DAvis 1951 Three cerebral systems affecting autonomic functions. Ibid., 14: 507-514.

Votaw, C. L., 1957 The hippocampus as an additional motor area. Anat. Rec., 127 (Suppl.): 382-383. 


\section{PLATE 1}

EXPLANATION OF FIGURES

4 Photomicrograph of a parasagittal section of the right hemisphere of monkey R2 with the degenerated fibers indicated by the arrow. $\times 2$.

5 Photomicrograph of the degenerated fibers indicated by the arrow in figure 4. $\times 37$.

6 Photomicrograph of a transverse section through the corpus eallosum of monkey R3 illustrating the course of the degenerated fibers. $\times 2.5$.

7 Photomicrograph of the degenerated fibers indicated by the arrow in 6 $\times 20$. 


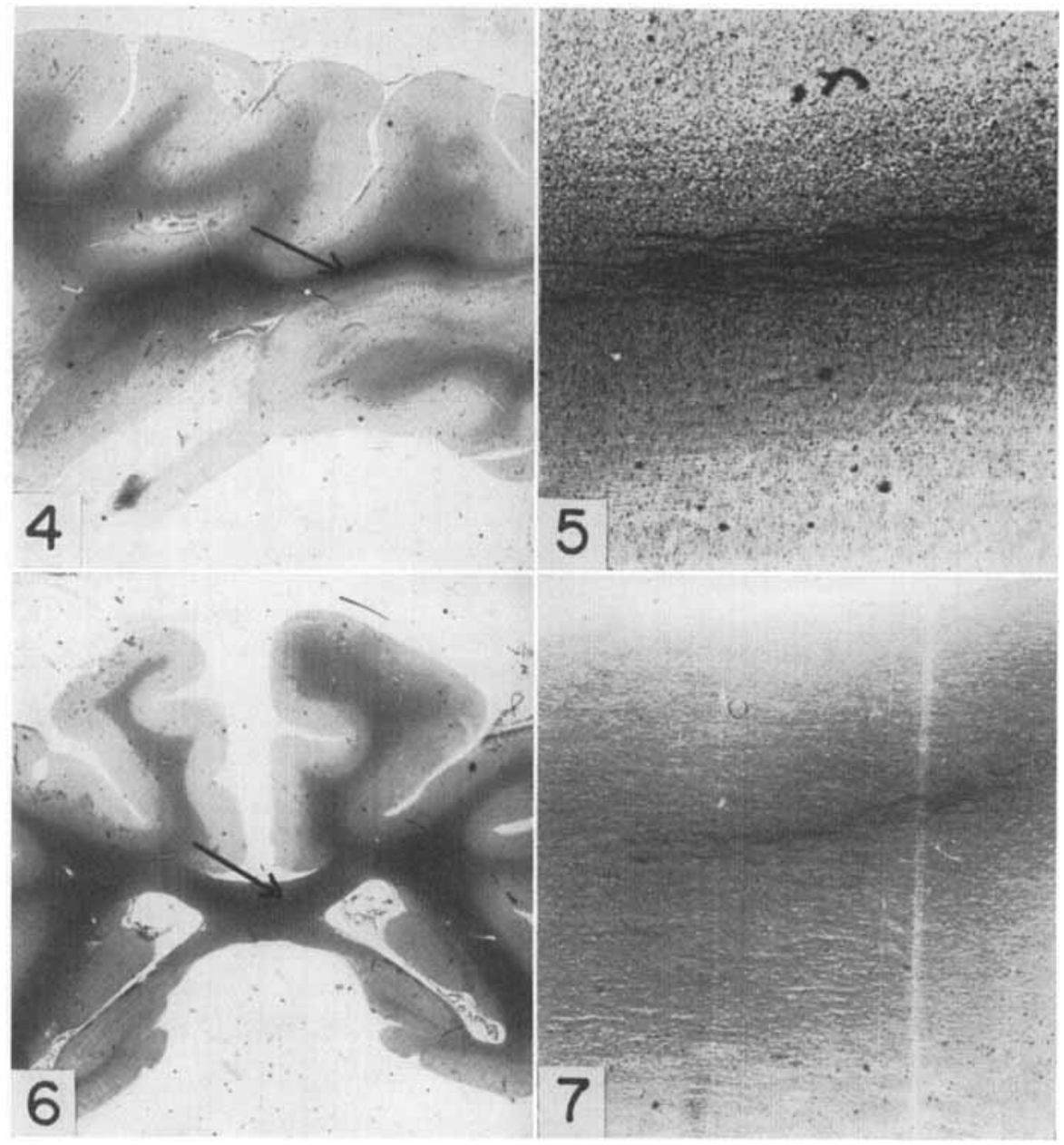


PLATE 2

EXPIANATION OF FIGURES

8 Photomicrograph of a transverse scetion through the lateral ventricles of monkey 124 . The degenerated fibors beneath the ventriclo are indicated by the arrow. $\times 2$.

9 Photomicrograph of the degenerated fibers indicated by the arrow in figure $8 . \times 37$.

10 Photomicrograph of a transverse section through the pons and midbrain of monkey $\mathrm{R} 4$. The area lateral to the red nucleus is indicated by the arrow. $\times 2$.

11. Photomicrograph of the degenerated fibers indicated by the arrow in figure 10. $\times 37$.

12 Photomicrograph of a transverse section through the pous and midbrain. The arrow indicates the internal capsule. $\times 2$.

13 Photomierograph of a transverse section through the internal capsule. The arrow indicates a fascicle of degenerating fibers, $\times 100$. 


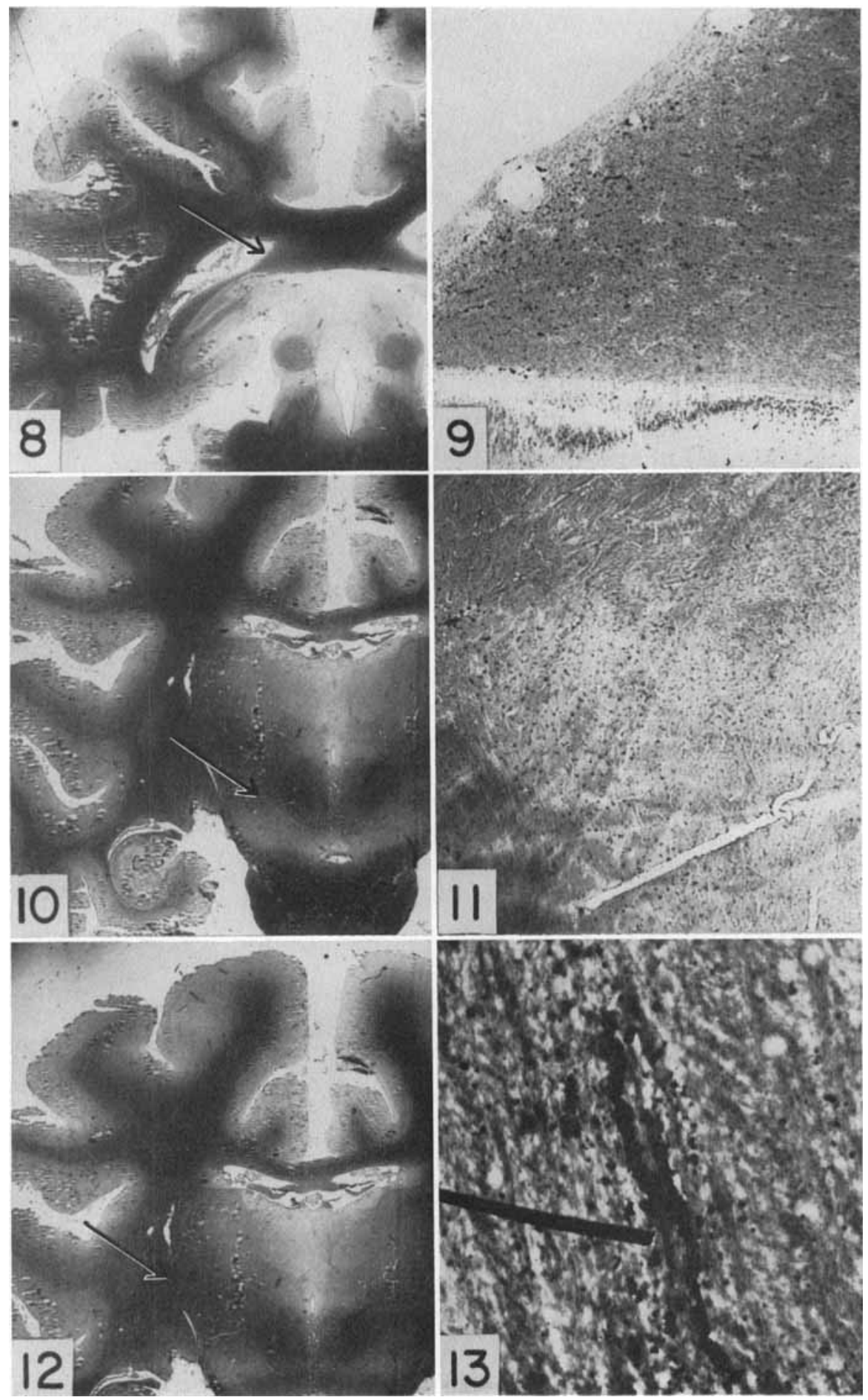

